PROCEEDINGS OF THE

AMERICAN MATHEMATICAL SOCIETY

Volume 131, Number 7, Pages 2109-2119

S 0002-9939(03)06807-2

Article electronically published on February 11, 2003

\title{
THE BANACH ALGEBRA INDUCED BY A DOUBLE CENTRALIZER
}

\author{
ETIENNE DESQUITH
}

(Communicated by David R. Larson)

\begin{abstract}
Given a Banach algebra $A, \mathrm{R}$. Larsen defined, in his book " $A n$ introduction to the theory of multipliers", a Banach algebra $A_{T}$ by means of a multiplier $T$ on $A$, and essentially used it in the case of a commutative semisimple Banach algebra $A$ to prove a result on multiplications which preserve regular maximal ideals. Here, we consider the analogue Banach algebra $\mathcal{A}_{R}$ induced by a bounded double centralizer $\langle L, R\rangle$ of a Banach algebra $A$. Then, our main concern is devoted to the relationships between $L, R$, and the algebras of bounded double centralizers $\mathcal{W}(A)$ and $\mathcal{W}\left(\mathcal{A}_{R}\right)$ of $A$ and $\mathcal{A}_{R}$, respectively. By removing the assumption of semisimplicity, we generalize some results proven by Larsen.
\end{abstract}

\section{Preliminaries}

Unless otherwise stated, we shall adopt throughout the sequel the following conventions: $A$ will be an arbitrary Banach algebra, and the Banach algebra of all (resp. bounded) linear operators on it will be denoted by $\mathcal{L}(A)$ (resp. $B(A)$ ). The maps $\lambda$ and $\mu$, such that $a \mapsto \lambda_{a}$ and $a \mapsto \mu_{a}$ for $a \in A$, are the usual left and right regular representations of $A$. Composition of mappings will be denoted by simple juxtaposition.

Definition 1.1. A left (resp. right) centralizer of $A$ is an element $L \in \mathcal{L}(A)$ (resp. $R \in \mathcal{L}(A)$ ), satisfying $L(a b)=L(a) b$ (resp. $R(a b)=a R(b), \forall a, b \in A$ ).

A double centralizer of $A$ is a pair $\langle L, R\rangle$ where $L$ (resp. $R$ ) is a left (resp. right) centralizer, and which together satisfy the following Double Centralizer Property (DC-Property):

$$
a L(b)=R(a) b \quad, \quad \forall a, b \in A .
$$

The algebra $\mathcal{W}(A)$ of bounded double centralizers of $A$ is the set of double centralizers with pointwise linear operations and with product and norm given by

$$
\begin{aligned}
\langle L, R\rangle\langle T, S\rangle= & \langle L T, S R\rangle, \quad\|\langle L, R\rangle\|=\operatorname{Max}\{\|L\| ;\|R\|\}, \\
& \forall\langle L, R\rangle,\langle T, S\rangle \in \mathcal{W}(A) .
\end{aligned}
$$

Relation (2) above ensures the automatic continuity of the elements $L$ and $R$ of any double centralizer $\langle L, R\rangle$ of $A$. For the definitions of a multiplier and of the

Received by the editors November 28, 2001 and, in revised form, February 4, 2002.

2000 Mathematics Subject Classification. Primary 47D30.

Key words and phrases. Annihilator, Banach algebra, double centralizer, multiplier.

This work was supported by the Abdus Salam ICTP Associateship scheme (Trieste/Italy). 
left annihilator $L A n(A)$, right annihilator $R A n(A)$ and annihilator $A n(A)$ of $A$, the reader should refer to [3] and [4]. $A$ is said to have no annihilators if $A n(A)=$ $\operatorname{LAn}(A)=R A n(A)=\{0\}$.

\section{The Banach algebra $\mathcal{A}_{R}$}

Proposition 2.1. Let $\langle L, R\rangle \in \mathcal{W}(A)$ such that $\|R\| \leq 1$, and define

$$
a \cdot b=R(a) b \quad, \quad \forall a, b \in A .
$$

(i) Relation (3) endows $A$ with a Banach algebra structure denoted by $\mathcal{A}_{R}$.

(ii) $R$ (resp. $L$ ) is a homomorphism from $\mathcal{A}_{R}$ onto the image $\operatorname{Im}(R)$ (resp. $\operatorname{Im}(L))$ of $R$ (resp. L).

(iii) If $A$ has no annihilator, or if $A$ admits a two-sided approximate identity, then the kernels of $L$ and $R$ satisfy $\operatorname{Ker}(R)=L A n\left(\mathcal{A}_{R}\right), \operatorname{Ker}(L)=R A n\left(\mathcal{A}_{R}\right)$, and hence $A n\left(\mathcal{A}_{R}\right)=\operatorname{Ker}(L) \cap \operatorname{Ker}(R)$.

Proof. ( $i$ ) and (ii) are obtained by straightforward computations. It is also easy to show the relations $R\left[L A n\left(\mathcal{A}_{R}\right)\right] \subset L A n(A)$ and $L\left[R A n\left(\mathcal{A}_{R}\right)\right] \subset R A n(A)$, from which (iii) follows, since $A$ is without annihilators.

Remark 2.2. It should be noticed that assuming $\|L\| \leq 1$, and defining the product in relation (3) as $a . b=a L(b), \forall a, b \in A$, gives the same algebra $\mathcal{A}_{R}$. Therefore, when dealing in the sequel with the algebra $\mathcal{A}_{R}$, we shall say that $\mathcal{A}_{R}$ is induced or defined by $\langle L, R\rangle$. Considering $\mathcal{A}_{R}$ as a left Banach module over itself, the map $R$ satisfying relation (3) is an example of a bounded module map from the module into the algebra (cf. [1]).

As an immediate consequence of Proposition 2.1 above, we have:

Corollary 2.3. Let $A$ have no annihilators. Then, for any $\langle L, R\rangle \in \mathcal{W}(A), L$ (resp. $R$ ) is one-to-one implies that $A n\left(\mathcal{A}_{R}\right)=\{0\}$. Moreover, $\mathcal{A}_{R}$ has no annihilators, if and only if $L$ and $R$ are both one-to-one.

Remark 2.4. If $A$ is commutative and without annihilators, then $\langle L, R\rangle$ is a multiplier $L=R=T$ and therefore $\mathcal{A}_{R}$ coincides with the algebra $A_{T}$ of $\mathrm{R}$. Larsen, and is also commutative.

For $\langle L, R\rangle$ defining $\mathcal{A}_{R}$, direct computations show that the condition $\langle T, S\rangle \in$ $\mathcal{W}\left(\mathcal{A}_{R}\right)$ implies $\langle L T, R S\rangle \in \mathcal{W}(A)$.

As noted by T. W. Palmer in [4, page 26, the set of left (resp. right) centralizers is contained in the commutant $\left(\mu_{A}\right)^{c}$ (resp. $\left.\left(\lambda_{A}\right)^{c}\right)$ of $\mu_{A}$ (resp. $\lambda_{A}$ ). Moreover, as pointed out in 2. page 775 , if $A$ has no annihilators, then given two arbitrary elements $\langle L, R\rangle$ and $\langle T, S\rangle$ of $\mathcal{W}(A)$, their components cross-commute, i.e. $T R=$ $R T$ and $L S=S L$. As a consequence, any $\langle L, R\rangle \in \mathcal{W}(A)$ satisfies $L R=R L$.

For every $\langle L, R\rangle \in \mathcal{W}(A)$, let $\operatorname{Im}(L)$ (resp. $\operatorname{Im}(R)$ ) denote the image of $L$ (resp. $R$ ), and define $I_{L R}=\operatorname{Im}(L) \cap \operatorname{Im}(R)$. Then we have:

Proposition 2.5. Let $A$ have no annihilators, and let $\langle L, R\rangle \in \mathcal{W}(A) \backslash\{0\}$. If $\overline{\operatorname{Im}(L)} \neq A$ and $\overline{\operatorname{Im}(R)} \neq A$, then each of $L$ and $R$ admits a nontrivial closed invariant subspace (the bar denotes the closure in the norm topology of $A$ ); moreover, $I_{L R}=\{0\} \quad$ implies that $L($ resp. $R)$ cannot be one-to-one. 
Proof. We shall write $L[\operatorname{Im}(R)]$ and $R[\operatorname{Im}(L)]$ to denote the restrictions of the maps $L$ and $R$, respectively, on the subspaces $\operatorname{Im}(R)$ and $\operatorname{Im}(L)$ of $A$. According to the above comments, the components of $\langle L, R\rangle \in \mathcal{W}(A)$ commute. Hence the following hold: $L[\operatorname{Im}(R)] \subset I_{L R}$ and $R[\operatorname{Im}(L)] \subset I_{L R}$, from which we derive $L[\overline{\operatorname{Im}(R)}] \subset \overline{\operatorname{Im}(R)}, R[\overline{\operatorname{Im}(L)}] \subset \overline{\operatorname{Im}(L)}$. Then, if the images of $L$ and $R$ are not dense in $A$, these inclusions prove that each of $L$ and $R$ admits a nontrivial closed invariant subspace. $L$ and $R$ being nonzero, the same inclusions imply that $L$ (resp. $R$ ) cannot be one-to-one if $I_{L R}=\{0\}$.

Theorem 2.6. Let $A$ and $\mathcal{A}_{R}$ be without annihilators and let $\langle L, R\rangle \in \mathcal{W}(A)$ be such that $\operatorname{Im}(L) \subset R[\operatorname{Im}(L)]$. Then, $R$ is bijective, and $R^{-1}$ is a right centralizer. The result remains clearly true if the roles of $R$ and $L$ are interchanged, and the right centralizer is replaced by the left centralizer.

Proof. We have, according to Corollary 2.3 and the proof of Proposition 2.5, $R[\operatorname{Im}(L)] \subset \operatorname{Im}(L)$, which, together with the assumption on $\operatorname{Im}(L)$ implies the equality $\operatorname{Im}(L)=R[\operatorname{Im}(L)]$. It then follows that $R$ is onto $A$, according to the following result: $L\left(x_{0}\right) \in R[\operatorname{Im}(L)] \Longrightarrow x_{0} \in \operatorname{Im}(R)$. Indeed, let us assume by contradiction that $x_{0} \notin \operatorname{Im}(R)$. Then, $L$ being one-to-one, we get $L\left(x_{0}\right) \neq L R(a), \forall a \in A$. But since $L$ commutes with $R$, it also follows that $L\left(x_{0}\right) \neq R L(a), \forall a \in A$, which means that $L\left(x_{0}\right) \notin R[\operatorname{Im}(L)]$. Hence, the desired result is established, and one easily checks that $R^{-1}$ is a right centralizer.

Let us define

$$
\begin{aligned}
& \mathcal{C}_{\ell}(A)=\{L \in B(A) ; \exists R \in B(A) ; \quad\langle L, R\rangle \in \mathcal{W}(A)\} ; \\
& \mathcal{C}_{r}(A)=\{S \in B(A) ; \exists T \in B(A) ; \quad\langle T, S\rangle \in \mathcal{W}(A)\} ; \\
& \Phi(\langle L, R\rangle)=L \quad, \quad \Psi(\langle L, R\rangle)=R \quad, \quad \forall\langle L, R\rangle \in \mathcal{W}(A) .
\end{aligned}
$$

Then we get:

Theorem 2.7. Let $A$ be a Banach algebra with an approximate identity bounded by one. Then $\mathcal{C}_{\ell}(A)$ and $\mathcal{C}_{r}(A)$ are Banach subalgebras of $B(A)$, and $\Phi$ is a faithful representation of $\mathcal{W}(A)$ on $A$, with $\Phi(\mathcal{W}(A))=\mathcal{C}_{\ell}(A)$ (resp. $\Psi$ is a one-to-one anti-homomorphism of $\mathcal{W}(A)$ into $B(A)$, with $\left.\Psi(\mathcal{W}(A))=\mathcal{C}_{r}(A)\right)$.

Proof. $\mathcal{C}_{\ell}(A)$ is clearly a linear subspace of $B(A)$. Moreover, if $L, T \in \mathcal{C}_{\ell}(A)$, then by definition, there exist $R, S \in B(A)$ such that $\langle L, R\rangle \in \mathcal{W}(A),\langle T, S\rangle \in \mathcal{W}(A)$, with $L=\Phi(\langle L, R\rangle)$ and $T=\Phi(\langle T, S\rangle)$. Hence, $\langle L, R\rangle\langle T, S\rangle=\langle L T, S R\rangle \in$ $\mathcal{W}(A)$, and therefore $L T=\Phi(\langle L T, S R\rangle) \in \mathcal{C}_{\ell}(A)$. So $\mathcal{C}_{\ell}(A)$ is a subalgebra of $B(A)$ and more precisely, a Banach algebra. Indeed, if $\left(L_{n}\right)_{n \geq 1}$ is a sequence in $\mathcal{C}_{\ell}(A)$ converging to some $L \in B(A)$, then $L$ is a left centralizer. Moreover, the corresponding sequence $\left(R_{n}\right)_{n \geq 1}$ in $B(A)$ such that $L_{n}=\Phi\left(\left\langle L_{n}, R_{n}\right\rangle, \forall n \geq 1\right.$, converges to some $R \in B(A)$. To see this, we proceed as follows: given $x \in A$, we let $x \mapsto \lambda_{x}$ be the left regular representation of $A$ onto itself, and for fixed $n, m \in \mathbb{N}$ and $a \in A$, we consider the map

$$
A \ni b \mapsto\left(R_{n}-R_{m}\right)(a) b=\lambda_{\left(R_{n}-R_{m}\right)(a)}(b) .
$$


Then we get

(8)

$$
\left\|\left(R_{n}-R_{m}\right)(a)\right\|=\left\|\lambda_{\left(R_{n}-R_{m}\right)(a)}\right\|=\operatorname{Sup}\left\{\left\|\left(R_{n}-R_{m}\right)(a)(b)\right\| ;\|b\| \leq 1\right\},
$$

so that the following holds:

(9)

$$
\left\|R_{n}-R_{m}\right\| \leq \operatorname{Sup}\left\{\operatorname{Sup}\left\{\left\|\left(R_{n}-R_{m}\right)(a)(b)\right\| ;\|b\| \leq 1\right\} ;\|a\| \leq 1\right\} .
$$

Since $\left\langle L_{n}, R_{n}\right\rangle,\left\langle L_{m}, R_{m}\right\rangle \in \mathcal{W}(A)$, we have, for each pair of elements $a, b \in A$,

$$
R_{n}(a) b=a L_{n}(b) \quad \text { and } \quad R_{m}(a) b=a L_{m}(b),
$$

and the equality (8) hence gives

$$
\begin{aligned}
\left\|R_{n}-R_{m}\right\| & \leq \operatorname{Sup}\left\{\|a\| \operatorname{Sup}\left\{\left\|L_{n}-L_{m}\right\|\|b\| ;\|b\| \leq 1\right\} ;\|a\| \leq 1\right\} \\
& \leq\left\|L_{n}-L_{m}\right\| .
\end{aligned}
$$

$\left(R_{n}\right)_{n \geq 1}$ is therefore a Cauchy sequence in $B(A)$, and hence converges to some $R \in B(A)$.

Now, for each $n \in \mathbb{N}$ and for all $a, b \in A$ the following relations are satisfied:

$$
\text { (i) } R_{n}(a b)=a R_{n}(b) \quad \text { and } \quad(i i) \quad R_{n}(a) b=a L_{n}(b) \text {, }
$$

and we get, taking the limits in equalities $(i)$ and $(i i)$ when $n$ tends to infinity: $R(a b)=a R(b)$ and $R(a) b=a L(b)$, that is, $R$ is a right centralizer and $\langle L, R\rangle \in$ $\mathcal{W}(A)$. Therefore $L=\Phi(\langle L, R\rangle) \in \mathcal{C}_{\ell}(A)$, and $\mathcal{C}_{\ell}(A)$ is hence norm-closed in $B(A)$. That $\mathcal{C}_{r}(A)$ is a Banach subalgebra of $B(A)$ is obtained by quite similar arguments as in the case of $\mathcal{C}_{\ell}(A)$. That $\Phi$ is a faithful representation of $\mathcal{W}(A)$ on $A$, and $\Psi$ a one-to-one anti-homomorphism of $\mathcal{W}(A)$ into $A$, are obtained by routine computations.

Given $R \in B(A)$, let $\{R\}^{c}=\{S \in B(A) ; R S=S R\}$ denote the commutant of $\{R\}$. According to the comments following Remark 2.4, if $\langle L, R\rangle \in \mathcal{W}(A)$ defines $\mathcal{A}_{R}$ and if $S \in\{R\}^{c}$ is such that $\langle T, S\rangle \in \mathcal{W}\left(\mathcal{A}_{R}\right)$, then $\langle L T, R S\rangle=\langle L T, S R\rangle \in$ $\mathcal{W}(A)$, and it is "tempting" in this situation to write $\langle L T, S R\rangle=\langle L, R\rangle\langle T, S\rangle$. But, the product in the right-hand side of this equation would not make sense, unless $\mathcal{W}(A)$ and $\mathcal{W}\left(\mathcal{A}_{R}\right)$ are subalgebras of some larger Banach algebra. We construct in what follows such an algebra.

Consider the set $\mathcal{D}_{P 0}(A)$ of all pairs $\langle L, R\rangle$ with $L \in B(A), R \in B(A)$, made into a linear algebra under pointwise linear operations and with the same product as in $\mathcal{W}(A)$. Then $\mathcal{D}_{P 0}(A)$ is a normed algebra when it is endowed with the same norm as in $\mathcal{W}(A)$. Now, since the elements of $\mathcal{D}_{P 0}(A)$ are not assumed to satisfy the $D C$-Property, $\mathcal{D}_{P 0}(A)$ may not be a Banach algebra. So we just have to consider the norm completion $\mathcal{D}_{P}(A)$ of $\mathcal{D}_{P 0}(A)$ to have our needed Banach algebra. The notation $\mathcal{D}_{P}(A)$, for the algebra just constructed, is motivated by the fact that we may think of its elements as bounded Double Pre-centralizers of A.

Proposition 2.8 below follows from the above comments and the previous results.

Proposition 2.8. $\mathcal{W}(A)$ and $\mathcal{W}\left(\mathcal{A}_{R}\right)$ are Banach subalgebras of the Banach algebra $\mathcal{D}_{P}(A)$, with nonvoid intersection, since $\langle L, R\rangle \in \mathcal{W}(A) \cap \mathcal{W}\left(\mathcal{A}_{R}\right)$. Moreover, $\langle T, S\rangle \in \mathcal{W}\left(\mathcal{A}_{R}\right)$ if and only if $\langle L T, R S\rangle \in \mathcal{W}(A)$. 
N.B. Throughout the remainder of the sequel, and unless otherwise stated, $A$ will always be a Banach algebra without annihilators and $\mathcal{A}_{R}$ will be the Banach algebra in Proposition 2.1, induced by some double centralizer $\langle L, R\rangle \in \mathcal{W}(A)$.

Given $a \in A$, let us set

$$
\begin{gathered}
\lambda_{a}^{R}(x)=R(a) x=\lambda_{R(a)}(x)=[(\lambda R)(a)](x), \quad \forall x \in A, \\
\mu_{a}^{R}(x)=R(x) a=x L(a)=[(\mu L)(a)](x), \quad \forall x \in A,
\end{gathered}
$$

and let $u_{R}$ be defined by $u_{R}(a)=\left\langle\lambda_{a}^{R}, \mu_{a}^{R}\right\rangle, \forall a \in \mathcal{A}_{R}$. Then we have:

Proposition 2.9. Let $\langle L, R\rangle \in \mathcal{W}(A)$ define $\mathcal{W}\left(\mathcal{A}_{R}\right)$. Then

(i) $u_{R}$ is the regular homomorphism of $\mathcal{A}_{R}$ into $\mathcal{W}\left(\mathcal{A}_{R}\right)$, and if there exists $z \in A$ such that $L(z)=R(z)$, we have in the algebra $\mathcal{D}_{P}(A)$ :

$$
\left\{\left\langle\lambda_{z}, \mu_{z}\right\rangle,\left\langle\lambda_{z}^{R}, \mu_{z}^{R}\right\rangle\right\} \subset \mathcal{W}(A) \cap \mathcal{W}\left(\mathcal{A}_{R}\right) .
$$

(ii) If $\langle L, R\rangle$ is a multiplier $(T=L=R)$, then $\mathcal{W}(A)$ is a Banach subalgebra of $\mathcal{W}\left(\mathcal{A}_{R}\right)$.

Proof. (i) Short computations using the definition of the product in $\mathcal{W}\left(\mathcal{A}_{R}\right)$ show that $a \mapsto u_{R}(a)=\left\langle\lambda_{a}^{R}, \mu_{a}^{R}\right\rangle$ is a homomorphism into $\mathcal{W}\left(\mathcal{A}_{R}\right)$ and that $\lambda_{a}^{R}$ and $\mu_{a}^{R}$ satisfy the DC-Property in $\mathcal{W}\left(\mathcal{A}_{R}\right)$. The regular homomorphism $u: a \mapsto\left\langle\lambda_{a}, \mu_{a}\right\rangle$, being from $A$ into $\mathcal{W}(A),\left\langle\lambda_{z}, \mu_{z}\right\rangle$ and $\left\langle\lambda_{R(z)}, \mu_{R(z)}\right\rangle$, belong to $\mathcal{W}(A)$, for each $z \in A$. Let us show that these two elements belong to $\mathcal{W}\left(\mathcal{A}_{R}\right)$, whenever $z \in A$ fulfills $L(z)=R(z)$. Indeed, for each $a \in A$, we have

$$
\begin{array}{ll}
x \cdot \lambda_{a}(y)=R(x) a y=x L(a) y, & \forall x, y \in A, \\
\mu_{a}(x) \cdot y=R(x a) y=x R(a) y, & \forall x, y \in A .
\end{array}
$$

Hence, if $z \in A$ satisfies $L(z)=R(z)$, and since $A$ is without annihilators, relations (15) and (16) above yield $\left\langle\lambda_{z}, \mu_{z}\right\rangle \in \mathcal{W}\left(\mathcal{A}_{R}\right)$. For $z$ with the above property, we have

$$
\begin{aligned}
x \cdot \lambda_{R(z)}(y) & =R(x) R(z) y=x L R(z) y=x R L(z) y \quad(L \text { and } R \text { commute }) \\
& =R(x L(z)) y=(x L(z)) \cdot y=(x R(z)) \cdot y \quad(L(z)=R(z)) \\
& =\mu_{R(z)}(x) \cdot y
\end{aligned}
$$

that is, $\left\langle\lambda_{R(z)}, \mu_{R(z)}\right\rangle=\left\langle\lambda_{z}^{R}, \mu_{z}^{R}\right\rangle \in \mathcal{W}\left(\mathcal{A}_{R}\right)$, and the desired result, $\left\langle\lambda_{z}^{R}, \mu_{z}^{R}\right\rangle \in$ $\mathcal{W}\left(\mathcal{A}_{R}\right) \cap \mathcal{W}(A)$, hence follows.

(ii) Let $\langle L, R\rangle \in \mathcal{W}(A)$ be such that $L=R$, so that $R$ satisfies $a R(b)=R(a) b$ for all $a, b \in A$. Then given any $\langle T, S\rangle \in \mathcal{W}(A)$, we have

$$
\begin{aligned}
a \cdot T(b) & =R(a) T(b)=a R(T(b))=R(a T(b)) \\
& =R(S(a) b)=R(S(a)) b=S(a) \cdot b
\end{aligned}
$$

which proves that $\langle T, S\rangle \in \mathcal{W}\left(\mathcal{A}_{R}\right)$. 
Remark 2.10. There exists a homomorphism $\varphi$ from $\mathcal{W}\left(\mathcal{A}_{R}\right)$ into $\mathcal{W}(A)$ which makes the following diagram commutative:

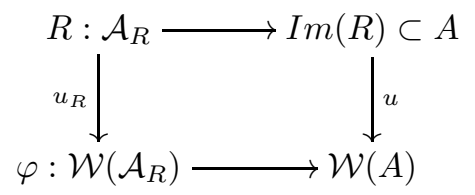

where $u$ and $u_{R}$ denote respectively the regular homomorphisms of $A$ into $\mathcal{W}(A)$ and of $\mathcal{A}_{R}$ into $\mathcal{W}\left(\mathcal{A}_{R}\right)$. Indeed, the homomorphisms $u R$ and $u_{R}$ are defined from $\mathcal{A}_{R}$, respectively into $\mathcal{W}(A)$ and $\mathcal{W}\left(\mathcal{A}_{R}\right)$, and satisfy $\operatorname{Ker}\left(u_{R}\right)=\{0\} \subset \operatorname{Ker}(u R)$. Then there exists a map $\varphi: u_{R}\left(\mathcal{A}_{R}\right) \longrightarrow \mathcal{W}(A)$ such that we have $\varphi u_{R}=u R$. Moreover, $\varphi$ is clearly a homomorphism from $u_{R}\left(\mathcal{A}_{R}\right)$ into $\mathcal{W}(A)$.

Under the hypotheses and notations of Remark 2.10, short computations lead to the following relations, for each $a \in A$, and each $\langle T, S\rangle \in \mathcal{W}\left(\mathcal{A}_{R}\right)$ :

$$
\begin{aligned}
& \langle T, S\rangle u_{R}(a)=\left\langle\lambda_{R T(a)}, \mu_{L T(a)}\right\rangle=\left\langle\lambda_{T(a)}^{R}, \mu_{T(a)}^{R}\right\rangle=u_{R}(T(a)), \\
& u_{R}(a)\langle T, S\rangle=\left\langle\lambda_{R S(a)}, \mu_{L S(a)}\right\rangle=\left\langle\lambda_{S(a)}^{R}, \mu_{S(a)}^{R}\right\rangle=u_{R}(S(a)) .
\end{aligned}
$$

Relations (17) and (18) show that for each $a \in A, \varphi$ is still defined on $\mathcal{W}\left(\mathcal{A}_{R}\right) u_{R}(a)$ and on $u_{R}(a) \mathcal{W}\left(\mathcal{A}_{R}\right)$ as follows:

$$
\begin{aligned}
& \varphi\left(\langle T, S\rangle u_{R}(a)\right)=\varphi\left(u_{R}(T(a))\right)=u[R T(a)]=\left\langle\lambda_{R T(a)}, \mu_{R T(a)}\right\rangle, \\
& \varphi\left(u_{R}(a)\langle T, S\rangle\right)=\varphi\left(u_{R}(S(a))\right)=u[R S(a)]=\left\langle\lambda_{R S(a)}, \mu_{R S(a)}\right\rangle .
\end{aligned}
$$

The next result gives the conditions under which $\varphi$ extends to the whole space $\mathcal{W}\left(\mathcal{A}_{R}\right)$.

Theorem 2.11. If for each $\langle T, S\rangle \in \mathcal{W}\left(\mathcal{A}_{R}\right)$ there exists $\langle P, Q\rangle \in \mathcal{W}(A)$ such that the system below is satisfied:

$$
\left\{\begin{array}{l}
P R=R T, \\
Q R=R S .
\end{array}\right.
$$

Then the map $\Theta: \mathcal{W}\left(\mathcal{A}_{R}\right) \longrightarrow \mathcal{W}(A)$, defined by $\Theta(\langle T, S\rangle)=\langle P, Q\rangle$, extends the homomorphism $\varphi: u_{R}(A) \longrightarrow \mathcal{W}(A)$. Furthermore, $\Theta$ is an isomorphism, provided $R$ is one-to-one.

Proof. If $\varphi$ extends to the whole of $\mathcal{W}\left(\mathcal{A}_{R}\right)$, then for each $a \in A$ and each $\langle T, S\rangle \in \mathcal{W}\left(\mathcal{A}_{R}\right)$, the element $\langle P, Q\rangle=\varphi(\langle T, S\rangle)$ would satisfy, according to (19) and (20),

$$
\begin{aligned}
\varphi\left(\langle T, S\rangle u_{R}(a)\right) & =\langle P, Q\rangle \varphi\left(u_{R}(a)\right)=\langle P, Q\rangle\left\langle\lambda_{R(a)}, \mu_{R(a)}\right\rangle \\
& =\left\langle\lambda_{R T(a)}, \mu_{R T(a)}\right\rangle, \\
\varphi\left(u_{R}(a)\langle T, S\rangle\right) & =\varphi\left(u_{R}(a)\right)\langle P, Q\rangle=\left\langle\lambda_{R(a)}, \mu_{R(a)}\right\rangle\langle P, Q\rangle \\
& =\left\langle\lambda_{R S(a)}, \mu_{R S(a)}\right\rangle .
\end{aligned}
$$


But we have

$$
\begin{aligned}
& \langle P, Q\rangle\left\langle\lambda_{R(a)}, \mu_{R(a)}\right\rangle=\left\langle P \lambda_{R(a)}, \mu_{R(a)} Q\right\rangle=\left\langle\lambda_{P R(a)}, \mu_{P R(a)}\right\rangle, \\
& \left\langle\lambda_{R(a)}, \mu_{R(a)}\right\rangle\langle P, Q\rangle=\left\langle\lambda_{R(a)} P, Q \mu_{R(a)}\right\rangle=\left\langle\lambda_{Q R(a)}, \mu_{Q R(a)}\right\rangle .
\end{aligned}
$$

Hence, if $\varphi$ extends to $\mathcal{W}\left(\mathcal{A}_{R}\right)$, then we must have

$$
\left\{\begin{array}{l}
\left\langle\lambda_{R T(a)}, \mu_{R T(a)}\right\rangle=\left\langle\lambda_{P R(a)}, \mu_{P R(a)}\right\rangle \\
\left\langle\lambda_{R S(a)}, \mu_{R S(a)}\right\rangle=\left\langle\lambda_{Q R(a)}, \mu_{Q R(a)}\right\rangle
\end{array}\right.
$$

from which the system $(*)$ follows. So, if for each $\langle T, S\rangle \in \mathcal{W}\left(\mathcal{A}_{R}\right)$ there exists $\langle P, Q\rangle \in \mathcal{W}(A)$ such that the system (*) is satisfied, we define the map $\Theta$ : $\mathcal{W}\left(\mathcal{A}_{R}\right) \longrightarrow \mathcal{W}(A)$ by

$$
\Theta(\langle T, S\rangle)=\langle P, Q\rangle .
$$

The restriction of $\Theta$ coincides with $\varphi$ on $u_{R}\left(A_{R}\right)$ since for each $u_{R}(a)=$ $\left\langle\lambda_{R(a)}, \mu_{R(a)}\right\rangle$, the element $\langle P, Q\rangle=\left\langle\lambda_{R(a)}, \mu_{R(a)}\right\rangle \in \mathcal{W}(A)$ clearly fulfills the system $(*)$.

We need to show that $\Theta$ as defined above is a homomorphism. So let $\langle T, S\rangle$ and $\left\langle T^{\prime}, S^{\prime}\right\rangle$ belong to $\mathcal{W}\left(\mathcal{A}_{R}\right)$, and let $\langle P, Q\rangle$ and $\left\langle P^{\prime}, S^{\prime}\right\rangle$ be elements of $\mathcal{W}(A)$ satisfying

$$
\Theta(\langle T, S\rangle)=\langle P, Q\rangle \quad \text { and } \quad \Theta\left(\left\langle T^{\prime}, S^{\prime}\right\rangle\right)=\left\langle P^{\prime}, Q^{\prime}\right\rangle .
$$

Then $\left\langle T T^{\prime}, S^{\prime} S\right\rangle \in \mathcal{W}\left(\mathcal{A}_{R}\right),\left\langle P P^{\prime}, Q^{\prime} Q\right\rangle \in \mathcal{W}\left(\mathcal{A}_{R}\right)$, and we have

$$
\begin{aligned}
& \left(P P^{\prime}\right) R=P\left(P^{\prime} R\right)=P\left(R T^{\prime}\right)=(P R) T^{\prime}=R\left(T T^{\prime}\right), \\
& \left(Q^{\prime} Q\right) R=Q^{\prime}(Q R)=Q^{\prime}(R S)=\left(Q^{\prime} R\right) S=R\left(S^{\prime} S\right) .
\end{aligned}
$$

So, $\left\langle P P^{\prime}, Q Q^{\prime}\right\rangle \in \mathcal{W}(A)$ fulfills the system $(*)$. Therefore, by definition of $\Theta$, we get

$$
\begin{aligned}
\Theta\left(\left\langle T T^{\prime}, S^{\prime} S\right\rangle\right) & =\left\langle P P^{\prime}, Q^{\prime} Q\right\rangle=\langle P, Q\rangle\left\langle P^{\prime}, Q^{\prime}\right\rangle \\
& =\Theta(\langle T, S\rangle) \Theta\left(\left\langle T^{\prime}, S^{\prime}\right\rangle\right)
\end{aligned}
$$

and $\Theta$ is hence a homomorphism from $\mathcal{W}\left(\mathcal{A}_{R}\right)$ into $\mathcal{W}(A)$, as desired.

Now, if $\langle T, S\rangle \in \mathcal{W}\left(\mathcal{A}_{R}\right)$ is such that $\langle P, Q\rangle=\Theta(\langle T, S\rangle)=0$, then by virtue of the system $(*)$, the following holds: $\operatorname{Im}(T) \cup \operatorname{Im}(S) \subset \operatorname{Ker}(R)$, and if $R$ is a one-to-one map, then $T$ and $S$ must be identically null.

We next deal with the uniqueness of the above extension of the homomorphism $\varphi$.

Theorem 2.12. Let us assume that there exists another extension $\Sigma$ of the homomorphism $\varphi$ to the entire $\mathcal{W}\left(\mathcal{A}_{R}\right)$. Then, $\Sigma$ "fulfills" the system (*), and in the particular case where $R$ is bijective, there exists a unique way of extending $\varphi$ to $\mathcal{W}\left(\mathcal{A}_{R}\right)$.

Proof. $\Sigma$ must coincide with $\varphi$ on the image $u_{R}\left(A_{R}\right)$ of the regular homomorphism from $\mathcal{A}_{R}$ into $\mathcal{W}\left(\mathcal{A}_{R}\right)$, that is,

$$
\Sigma\left(u_{R}(a)\right)=\Sigma\left(\left\langle\lambda_{R(a)}, \mu_{L(a)}\right\rangle\right)=\left\langle\lambda_{R(a)}, \mu_{R(a)}\right\rangle,
$$


and since $u_{R}(A)$ is a two-sided ideal in $\mathcal{W}\left(\mathcal{A}_{R}\right), \Sigma$ must also satisfy

$$
\begin{aligned}
& \Sigma\left(\langle T, S\rangle u_{R}(a)\right)=\Sigma\left(\left\langle\lambda_{R T(a)}, \mu_{L T(a)}\right\rangle\right)=\left\langle\lambda_{R T(a)}, \mu_{R T(a)}\right\rangle, \\
& \Sigma\left(u_{R}(a)\langle T, S\rangle\right)=\Sigma\left(\left\langle\lambda_{R S(a)}, \mu_{L S(a)}\right\rangle\right)=\left\langle\lambda_{R S(a)}, \mu_{R S(a)}\right\rangle .
\end{aligned}
$$

So, if $\Sigma(\langle T, S\rangle)=\langle U, V\rangle$, then we must have

$$
\begin{aligned}
\Sigma\left(\langle T, S\rangle u_{R}(a)\right) & =\langle U, V\rangle\left\langle\lambda_{R(a)}, \mu_{R(a)}\right\rangle=\left\langle\lambda_{U R(a)}, \mu_{U R(a)}\right\rangle \\
& =\left\langle\lambda_{R T(a)}, \mu_{R T(a)}\right\rangle \\
\Sigma\left(u_{R}(a)\langle T, S\rangle\right) & =\left\langle\lambda_{R(a)}, \mu_{R(a)}\right\rangle\langle U, V\rangle=\left\langle\lambda_{V R(a)}, \mu_{V R(a)}\right\rangle \\
& =\left\langle\lambda_{R S(a)}, \mu_{R S(a)}\right\rangle .
\end{aligned}
$$

The above relations show that $\Sigma(\langle T, S\rangle)=\langle U, V\rangle$ satisfies the system $(*)$ as well, and therefore the following system (**) is also fulfilled by $\langle P, Q\rangle$ and $\langle U, V\rangle$ :

$$
\left\{\begin{array}{l}
P R=U R \\
Q R=V R
\end{array}\right.
$$

But the conditions in $(* *)$ above may also be interpreted in the following way, in terms of restrictions of maps: $\left.P\right|_{\operatorname{Im}(R)}=\left.U\right|_{\operatorname{Im}(R)}$ and $\left.Q\right|_{\operatorname{Im}(R)}=\left.V\right|_{\operatorname{Im}(R)}$, which, in the case where $R$ is bijective (and hence onto $A$ ), gives

$$
\Theta(\langle T, S\rangle)=\langle P, Q\rangle=\langle U, V\rangle=\Sigma(\langle T, S\rangle),
$$

that is, $\Theta=\Sigma$, which proves the uniqueness of the extension of $\varphi$. In such a case where the map $R$ is bijective, $R$ admits a well-defined inverse $R^{-1} \in B(A)$ which is a right centralizer, and therefore, the system (*) gives, for $\langle P, Q\rangle=\Theta(\langle T, S\rangle)$,

$$
P=R T R^{-1} \text { and } Q=R S R^{-1},
$$

which completes the proof.

Let us denote by $W^{R}(A)$, the normed algebra of bounded double centralizers of $\operatorname{Im}(R) \subseteq A$. Then it is clear that $\mathcal{W}(A) \subseteq W^{R}(A)$, and that equality holds whenever $R$ is onto $A$. The next result links $W^{R}(A)$ with the space of elements $P, Q \in B(A)$ which satisfy the system (*) with the elements of $\mathcal{W}\left(\mathcal{A}_{R}\right)$.

Proposition 2.13. Let $P, Q \in B(A)$ satisfy the system $(*)$ with some $\langle T, S\rangle \in$ $\mathcal{W}\left(\mathcal{A}_{R}\right)$. Then $\langle P, Q\rangle \in W^{R}(A)$.

Proof. For all $a, b \in \operatorname{Im}(R)$, we have $\alpha, \beta \in A$, such that $a=R(\alpha) ; b=R(\beta)$, and then, for each $\langle P, Q\rangle \in B(A)$ satisfying the system $(*)$ with some $\langle T, S\rangle \in \mathcal{W}\left(\mathcal{A}_{R}\right)$, the following holds:

$$
\begin{aligned}
a P(b) & =R(\alpha)(P R)(\beta)=R(\alpha)(R T)(\beta) \quad(\text { since }(*) \text { holds }) \\
& =R(R(\alpha) T(\beta)) \\
& =R(\alpha \cdot T(\beta))=R(S(\alpha) \cdot \beta) \quad\left(\text { since }\langle T, S\rangle \in \mathcal{W}\left(\mathcal{A}_{R}\right)\right) \\
& =R(R S(\alpha) \beta) \\
& =R S(\alpha) R(\beta)=Q R(\alpha) R(\beta) \quad \text { (since }(*) \text { holds) } \\
& =Q(a) b,
\end{aligned}
$$

which is the DC-Property in $W^{R}(A)$, and the proof is complete.

As is well known, the theory of double centralizers is a helpful device in the study of extensions of algebras. Let us recall the following definition (cf. [4], pp. 33 - 34). 
Definition 2.14. Let $A$ and $B$ be Banach algebras. An extension of $A$ by $C$ is a short exact sequence $0 \longrightarrow A \stackrel{\rho}{\rightarrow} B \stackrel{\psi}{\rightarrow} \quad C \longrightarrow 0$ of Banach algebras. The extension is called a semidirect product if there is a continuous homomorphism $\chi: C \longrightarrow B$, such that $\psi \chi \chi$ is the identity map on $C$. The short exact sequence is then said to split, with splitting homomorphism $\chi$.

Theorem 2.15. Let $A$ be a Banach algebra with a left approximate identity bounded by one. Let $\mathcal{A}_{R}$ be induced by $\langle L, R\rangle \in \mathcal{W}(A)$ with $R$ bijective. Then there exists an extension of $A$ by $\mathcal{W}\left(\mathcal{A}_{R}\right)$ :

$$
0 \longrightarrow A \stackrel{\rho}{\rightarrow} \quad B \stackrel{\psi}{\rightarrow} \mathcal{W}\left(\mathcal{A}_{R}\right) \longrightarrow 0
$$

and a continuous homomorphism $\Theta: B \longrightarrow \mathcal{W}(A)$, such that the following diagram commutes:

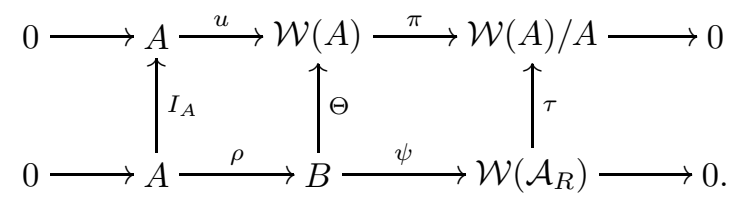

This extension, which is unique up to equivalence, is moreover a semidirect product.

Proof. Identifying $A$ with $u(A)$ under the regular homomorphism $u$ of $A$ into $\mathcal{W}(A)$, consider the short exact sequence $0 \longrightarrow A \stackrel{u}{\rightarrow} \mathcal{W}(A) \stackrel{\pi}{\rightarrow} \quad C \longrightarrow 0$, where $\pi$ is the natural map. Since $R$ is bijective, Theorem 2.12 asserts that $\Theta$ is a homeomorphic isomorphism of $\mathcal{W}\left(\mathcal{A}_{R}\right)$ onto $\mathcal{W}(A)$. The map $\tau: \mathcal{W}\left(\mathcal{A}_{R}\right) \longrightarrow$ $\mathcal{W}(A) / A$, defined by

$$
\tau(\langle T, S\rangle)=\Theta(\langle T, S\rangle)+A \quad, \quad \forall\langle T, S\rangle \in \mathcal{W}\left(\mathcal{A}_{R}\right),
$$

is hence a continuous homomorphism satisfying: $\tau=\pi \Theta$. Then, the hypotheses of Theorem 1.2.10 in [4, page 34, are satisfied, and its conclusion provides the existence of the extension of $A$ given by relation (30), and unique up to equivalence. Furthermore, in virtue of Theorem 1.2.11 in [4, page 35, the existence of $\tau$ satisfying $\tau=\pi \Theta$ shows that the extension is a semidirect product.

Now, let us assume that $A$ is commutative, and let us denote by $\mathcal{M}(A)$ the algebra of multipliers of $A$, which necessarily coincides with $\mathcal{W}(A)$. In [3], Corollary 1.3.1, page 23, R. Larsen proved that for a commutative semisimple Banach algebra $A$, the following equality holds: $\mathcal{M}(A)=\mathcal{M}\left(\mathcal{A}_{R}\right)$. In what follows, we still obtain the same result when the assumption of semisimplicity on $A$ is removed. We shall need the following:

Lemma 2.16. Let $A$ be a commutative Banach algebra and let $R \in \mathcal{M}(A)$. Then the following conditions are equivalent:

(i) $\mathcal{A}_{R}$ has no annnihilators;

(ii) A has no annihilators and $R$ is one-to-one.

Proof. Assume that $(i)$ is fulfilled, and let $a \in A$ be given. Then, $A$ being commutative and $R$ being a multiplier of $A$, we get for all $x \in A$ :

$$
R(x a)=R(a x)=R(a) x=a \cdot x=x \cdot a \quad\left(\mathcal{A}_{R} \text { is commutative }\right),
$$


which, since $\mathcal{A}_{R}$ is without annihilators, implies that $A$ has no annihilators. Moreover, due to Corollary 2.3, $R$ is one-to-one, if $\mathcal{A}_{R}$ has no annihilators. Hence (i) implies $(i i)$.

Conversely if (ii) is satisfied, then Corollary 2.3 again implies that $\mathcal{A}_{R}$ has no annihilators, and the equivalence of $(i)$ and $(i i)$ is therefore established.

We can now prove:

Theorem 2.17. Let $A$ be a commutative Banach algebra and let $R \in \mathcal{M}(A)$. Then $\mathcal{M}(A)=\mathcal{M}\left(\mathcal{A}_{R}\right)$ if and only if one of the equivalent conditions in Lemma 2.16 is satisfied.

Proof. We assume that condition $(i)$ of Lemma 2.16 holds. Let $T \in \mathcal{M}(A)$; then for all $a, b \in A$, we get $T(a \cdot b)=T[R(a) b]=R(a) T(b)=a \cdot T(b)$ and

$$
\begin{aligned}
T(a . b) & =R(a) T(b)=T(b) R(a)=R[T(b) a]=R[b T(a)] \\
& =R(T(a) b)=R[T(a)] b=T(a) . b,
\end{aligned}
$$

which means that $T \in \mathcal{M}(A)$, and consequently, $\mathcal{M}(A) \subseteq \mathcal{M}\left(\mathcal{A}_{R}\right)$.

Conversely, let $T \in \mathcal{M}\left(\mathcal{A}_{R}\right)$. Then, for all $a, b, x \in A$, we get on one hand

$$
\begin{aligned}
R(x) T(a b) & =x \cdot T(a b)=T(x) \cdot a b=a b T(x)=R(a b) T(x) \\
& =[R(a) b] T(x)=R(a) T(x) b=[a \cdot T(x)] b=[T(a) \cdot x] b \\
& =(R[T(a)] x)=T(a) R(x) b=R(x)[T(a) b]
\end{aligned}
$$

which implies, since $\mathcal{A}_{R}$ is without annihilators, $T(a b)=T(a) b$.

On the other hand, let $a, b, x \in A$; then

$$
R(x) T(a b)=R(a b) T(x)=a[b \cdot T(x)]=a[T(b) R(x)]=R(x)[a T(b)],
$$

and once again, the fact that $\mathcal{A}_{R}$ is without annihilators, leads to $T(a b)=a T(b)$. Therefore, $T \in \mathcal{M}(A)$, and this provides the reverse inclusion $\mathcal{M}\left(\mathcal{A}_{R}\right) \subseteq \mathcal{M}(A)$, and hence the desired equality $\mathcal{M}\left(\mathcal{A}_{R}\right)=\mathcal{M}(A)$.

The following result also appears in [3], Corollary 1.3.2, page 23:

Proposition 2.18. Let $A$ be a commutative semisimple Banach algebra which admits factorization, i.e.: For every $a \in A$, there exist $x, y \in A$, such that $a=x y$. Then the following conditions are equivalent:

(i) $A_{T}$ admits factorization;

(ii) $T$ is invertible.

Dropping the assumption of semisimplicity on $A$, we can prove the following:

Theorem 2.19. Let $A$ be a commutative Banach algebra which admits factorization, and let $R \in \mathcal{M}(A)$ be such that $\mathcal{A}_{R}$ is without annihilators. Then $\mathcal{A}_{R}$ admits factorization, if and only if $R$ is invertible.

Proof. Assume that $\mathcal{A}_{R}$ admits factorization. Then, since $\mathcal{A}_{R}$ is without annihilators, $R$ is one-to-one, according to Lemma 2.16. Moreover, since $\mathcal{A}_{R}$ admits factorization, for each $a \in \mathcal{A}_{R}$ there exist $\alpha, \beta \in \mathcal{A}_{R}$, such that $a=\alpha \cdot \beta=$ $R(\alpha) \beta=R(\alpha \beta)$, which means that $R$ is onto $A$, whence $R$ is bijective and $R^{-1}$ exists.

Conversely, let $R$ be invertible; then $R$ is one-to-one, and since it is also onto $A$, we get for each $a \in A$ some $\alpha \in \mathcal{A}_{R}$ satisfying $a=R(\alpha)$. But, $A$ admits factorization, and therefore $\alpha$ has the following decomposition: $\alpha=u v, u, v \in A$. 
Hence we get $a=R(\alpha)=R(u v)=R(u) v=u \cdot v$, so that $\mathcal{A}_{R}$ also admits factorization, and the proof is complete.

Coming back to the general case where $A$ is neither commutative nor semisimple, we give the analogous version of Theorem 2.17, when the multiplier algebra is replaced by the double centralizer algebra, as follows:

Theorem 2.20. Let $A$ be without annihilators, and let $\langle L, R\rangle \in \mathcal{W}(A)$ define $\mathcal{A}_{R}$ and be such that $R$ is bijective. Then $\mathcal{W}\left(\mathcal{A}_{R}\right)$ and $\mathcal{W}(A)$ are homeomorphically isomorphic.

Proof. According to Theorems 2.11 and 2.12, there exists a homomorphism $\Theta$ which extends in a unique way the homomorphism $\varphi$ constructed in Remark 2.10 to the algebra $\mathcal{W}\left(\mathcal{A}_{R}\right)$. Moreover, according to Theorem 2.11, each element $\langle T, S\rangle \in$ $\operatorname{Ker}(\Theta)$ satisfies $\operatorname{Im}(T) \cup \operatorname{Im}(S) \subset \operatorname{Ker}(R)$. So, if $R$ is bijective and hence one-to-one, $\operatorname{Ker}(R)=\{0\}$, and $\Theta$ is also one-to-one. Therefore, $\Theta$ is a bijection of $\mathcal{W}\left(\mathcal{A}_{R}\right)$ onto $\mathcal{W}(A)$. But, according to relation (29), the positive constant $K_{R}=\left\|R^{-1}\right\|\|R\|$ (depending only on $R$ ) satisfies $\|\Theta(\langle T, S\rangle)\| \leq$ $K_{R}\|\langle T, S\rangle\| ; \forall\langle T, S\rangle \in \mathcal{W}\left(\mathcal{A}_{R}\right)$, so that $\Theta$ is a bounded linear map from $\mathcal{W}\left(\mathcal{A}_{R}\right)$ onto the Banach space $\mathcal{W}(A)$, that is, a homeomorphism, and the proof is complete.

\section{REFERENCES}

1. Desquith, E.: Banach algebras associated to bounded module maps; Preprint ICTP $N^{0}$ IC 98/194; (1998).

2. Doran, R.S. \& Fell, J.M.G.: Representations of $*$-algebras, locally compact groups, and Banach $*$-algebraic Bundles, Vol. 2; Academic Press (1988). MR 90c: 46002

3. Larsen, R.: Introduction to the theory of multipliers; Springer-Verlag (1971). MR 55:8695

4. Palmer, T.W.: Banach algebras and the general theory of Banach*-algebras, Vol. I: Algebras and Banach algebras; Cambridge University Press (1994). MR 95c:46002

Institut de Recherches Mathématiques (IRMA), 08 BP 2030 Abidjan 08, Ivory Coast E-mail address: desquith@hotmail.com 